\title{
Ultrashort electric pulse induced changes in cellular dielectric properties
}

\author{
Allen L. Garner ${ }^{\mathrm{a}, \mathrm{b}, *}$, George Chen ${ }^{\mathrm{c}}$, Nianyong Chen ${ }^{\mathrm{b}, 1}$, Viswanadham Sridhara ${ }^{\mathrm{d}, 2}$, \\ Juergen F. Kolb ${ }^{\text {b,d }}$, R. James Swanson ${ }^{\text {b,e }}$, Stephen J. Beebe ${ }^{\text {b,f }}$, Ravindra P. Joshi ${ }^{\text {b,d }}$, \\ Karl H. Schoenbach ${ }^{b, d}$ \\ ${ }^{a}$ Bioelectromagnetism Laboratory, Department of Nuclear Engineering and Radiological Sciences, University of Michigan, Ann Arbor, MI 48109, USA \\ ${ }^{\mathrm{b}}$ Frank Reidy Research Center for Bioelectrics, Old Dominion University, Norfolk, VA 23510, USA \\ ${ }^{c}$ School of Electronics and Computer Science, University of Southampton, Southampton So17 1bj, UK \\ ${ }^{d}$ Department of Electrical and Computer Engineering, Old Dominion University, Norfolk, VA 23529, USA \\ ${ }^{\mathrm{e}}$ Department of Biological Sciences, Old Dominion University, Norfolk, VA 23529, USA \\ ${ }^{\mathrm{f}}$ Department of Physiological Sciences, Eastern Virginia Medical School, Norfolk, VA 23510, USA
}

Received 22 July 2007

Available online 7 August 2007

\begin{abstract}
The interaction of nanosecond duration pulsed electric fields (nsPEFs) with biological cells, and the models describing this behavior, depend critically on the electrical properties of the cells being pulsed. Here, we used time domain dielectric spectroscopy to measure the dielectric properties of Jurkat cells, a malignant human T-cell line, before and after exposure to five $10 \mathrm{~ns}, 150 \mathrm{kV} / \mathrm{cm}$ electrical pulses. The cytoplasm and nucleoplasm conductivities decreased dramatically following pulsing, corresponding to previously observed rises in cell suspension conductivity. This suggests that electropermeabilization occurred, resulting in ion transport from the cell's interior to the exterior. A delayed decrease in cell membrane conductivity after the nsPEFs possibly suggests long-term ion channel damage or use dependence due to repeated membrane charging and discharging. This data could be used in models describing the phenomena at work. (C) 2007 Elsevier Inc. All rights reserved.
\end{abstract}

Keywords: Dielectric spectroscopy; Nanosecond pulsed electric fields; Electroporation; Permeabilization; Plasma membrane; Time domain; Intracellular manipulation; Ultrashort pulses

The permeability of the cell membrane to external molecules can be dramatically increased by applying pulsed electric fields (PEFs) above a threshold voltage [1]. The mechanism behind this electropermeabilization is electroporation, or pore formation in the cell membrane, and usually occurs for PEFs with electric fields on the order of a

\footnotetext{
* Corresponding author. Present address: GE Global Research Center, Niskayuna, NY 12309, USA.

E-mail addresses: garner@ge.com (A.L. Garner),kschoenb@odu.edu (K.H. Schoenbach).

${ }^{1}$ Present address: Department of Medicine-Pulmonary and Critical Care, University of Virginia School of Medicine, Charlottesville, VA 22908, USA.

${ }^{2}$ Present address: National Center for Biotechnology Information, National Library of Medicine, National Institutes of Health, Bethesda, MD 20892, USA.
}

few $\mathrm{kV} / \mathrm{cm}$ and pulse durations on the order of 0.1 $10 \mathrm{~ms}$. With the appropriate combination of these parameters, electroporation is irreversible and the membrane breaks down, which is desirable for applications ranging from bacterial decontamination to food processing [2]. Many applications require temporarily opening the cell membrane to normally impermeant molecules, such as electrochemotherapy and gene therapy [3]. Applying pulses with higher field strength $(50-300 \mathrm{kV} / \mathrm{cm})$ and shorter duration (10-300 ns) do not fully charge the cell membrane and instead interact primarily with the membranes of intracellular organelles [4]. These nanosecond duration PEFs (nsPEFs) cause intracellular calcium release $[5,6]$ and apoptosis-induction in cell suspensions and in vivo tumors $[5,7,8]$. To better understand the mechanisms involved, attempts to develop mathematical models are underway 
[9]. These models depend on the electrical properties (e.g. capacitance and resistance) of the biological cells studied, typically HL-60 and Jurkat cell lines, which are not generally included in the literature [10].

We used time domain dielectric spectroscopy (TDDS) to determine the behavior of these parameters after applying PEFs to Jurkat cells. TDDS enables us to nonintrusively determine the electrical properties of the cell membrane, cytoplasm, nucleus, and nucleoplasm according to the twoshell model of the eukaryotic cell, shown in Fig. 1 [10-12]. We note that this model has also been referred to as a "three-shell model" [13]; however, we follow the two-shell nomenclature Feldman et al. TDDS can rapidly obtain dielectric spectra over a wide frequency range with uncertainties on the order $3-5 \%$ and $5-7 \%$ for the real and imaginary components of the permittivity [14], which are similar to frequency domain systems [15]. We previously used TDDS to study PEF-induced changes in HL-60 suspension conductivity due to single microsecond or nanosecond PEFs [16] and multiple nsPEFs [17]. In this communication, we extend our previous TDDS analysis of cell suspensions exposed to PEFs to include a full analysis of the temporal behavior of the electrical properties of Jurkat cells.

\section{Materials and methods}

Jurkat cell preparation. We used the Jurkat cell line, derived from human T-cell leukemia (America Type Culture Collection, Manassas, VA). The cells were cultured in RPMI-1640 Medium (ATCC), supplemented with $10 \%$ fetal bovine serum and $2 \%$ penicillin/streptomycin. We verified that Jurkat cell line viability exceeded $90 \%$ by using $0.4 \%$ trypan blue (Sigma). The cultured Jurkat cells were centrifuged for $5 \mathrm{~min}$ at approximately $200 \mathrm{~g}$ at room temperature and washed three times with a low-conductivity sucrose/glucose buffer ( $229 \mathrm{mM}$ sucrose, $16 \mathrm{mM}$ glucose, $1 \mu \mathrm{M} \mathrm{CaCl}$, and $5 \mathrm{mM} \mathrm{Na}_{2} \mathrm{HPO}_{4}$ ) in triple distilled water at $\mathrm{pH} 7.4$
$[10,16,17]$. This buffer was selected to minimize electrode polarization, or the formation of a parasitic capacitance layer near the measurement electrodes, while maintaining isotonic osmotic-pressure $(280 \mathrm{mOsmol})$ and cell morphology over the measurement period [10]. The cells were then adjusted to an $8 \%$ volume fraction.

nsPEF application. After preparation, the cell suspensions were placed in Gene Pulser cuvettes (BioRad) with an electrode distance of $1 \mathrm{~mm}$. A Blumlein pulse generator with an impedance of $10 \Omega$ provided $10 \mathrm{~ns}$ electrical pulses with a rise- and fall-time of 1-2 ns, limited by the employed high-pressure $\mathrm{SF}_{6}$ spark gap switch $[16,18]$. The Blumlein generator consists of two transmission lines with equal length and impedance in series with the load impedance matching the overall impedance of the two transmission lines, $2 Z_{0}$. More details can be found elsewhere [18].

The low conductivity buffer in these experiments had a higher resistance $(\sim 110 \Omega)$ than the HBSS-based buffers used in most nsPEF experiments $(\sim 10 \Omega)$, so we used a matching resistor to obtain an appropriate square pulse [16]. Each sample was exposed to a train of five $10 \mathrm{~ns}$ pulses at an electric field of $150 \mathrm{kV} / \mathrm{cm}$. The repetition rate of the pulses was limited by the charging time of the capacitor, and was typically approximately $10 \mathrm{~s}$.

TDDS measurement system. We used a Time Domain Spectrometer (IDC Expertise, UK), as shown in Fig. 1 [14]. TDDS is based on transmission line theory in the time domain [14]. A low voltage $(\sim 200 \mathrm{mV})$, rapidly increasing $(40-50 \mathrm{ps})$ voltage step arrives at a sampling head, where the signal reflected from the dielectric sample (cell suspension) is also measured. TDDS uses the lumped capacitance approximation [14], which considers the sample holder as a capacitor with a complex permittivity that is determined by comparing the reflected signal of the sample to two standards (open and short circuit loads). We used a thermostat (Julabo, US) to maintain the temperature of the sample holder at $25^{\circ} \mathrm{C}$ to be consistent with past experiments $[10,17,18]$.

Using data analysis software (IDC Expertise), we performed nonuniform sampling of a $5 \mu$ s time window to obtain the dielectric spectrum of the cell suspension and estimate and remove the effects of electrode polarization and DC conductivity $[10,17,18]$. We used a single-exponent model to correct for electrode polarization in the coaxial sample cell [19]. Determining and removing the conductivity of the suspension from the residual charge and performing a Fourier Transform of the remaining signal yields the real and imaginary components of permittivity of the cell suspension.

Two-shell model for eukaryotic cells. At this point, we applied the twoshell model of the cell, shown in Fig 1 and discussed in more detail else-

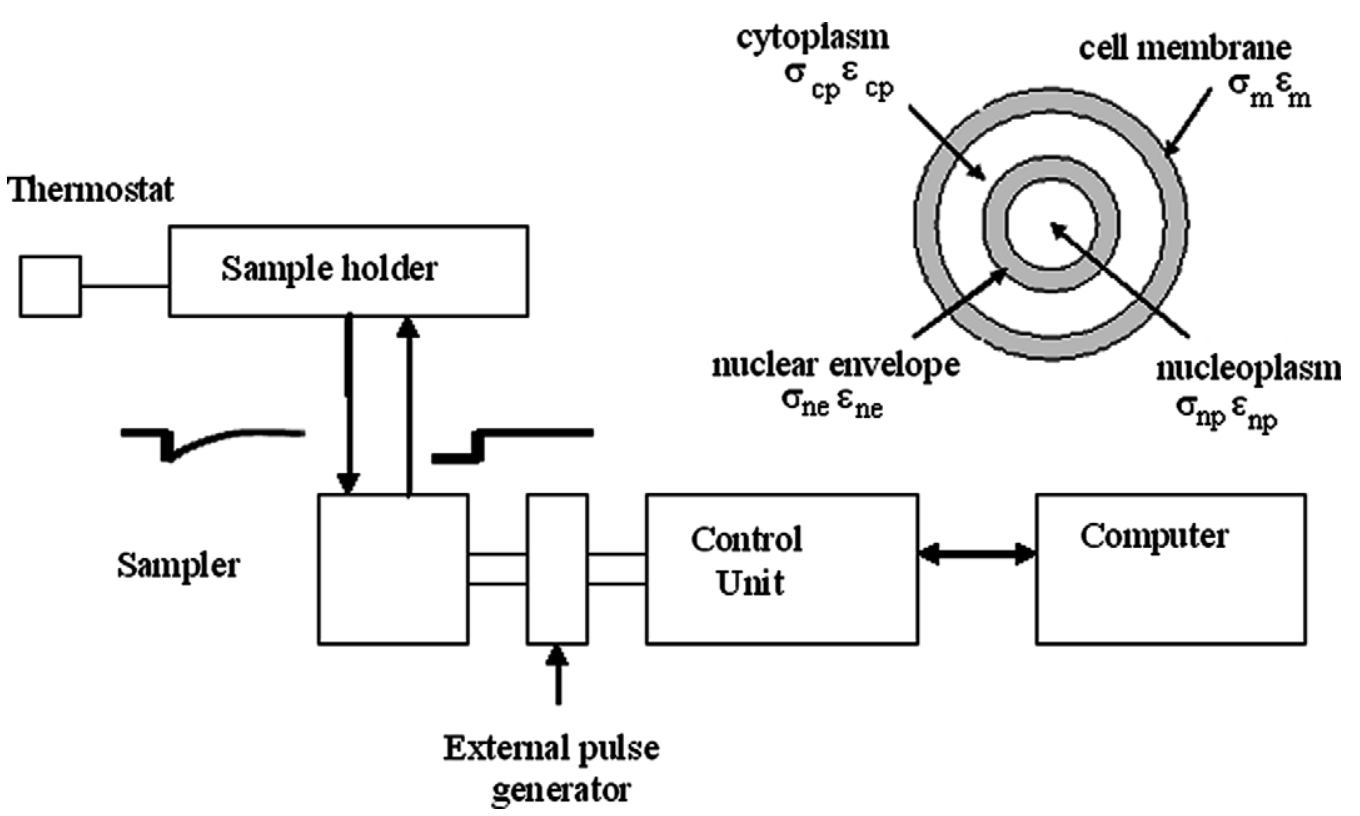

\section{Thermostat}

Fig. 1. Block diagram of the TDDS system, including shapes of the incident (left) and reflected (right) voltage signals and the two-shell model of a lymphocyte, where $\varepsilon$ is the permittivity and $\sigma$ is conductivity [11]. 
where [10]. In addition to these eight dielectric parameters, the model requires three geometric parameters. Only 6 of these 11 parameters can be solved for using the two-shell model, while the other parameters must be determined independently and fixed [10]. We set the cell membrane thickness $(7 \mathrm{~nm})$, nuclear envelope thickness $(14 \mathrm{~nm})$, nuclear volume fraction $(0.70)$, cytoplasm permittivity $\left(\varepsilon_{\mathrm{cp}}=60\right)$, and nucleoplasm permittivity $\left(\varepsilon_{\mathrm{np}}=120\right)[10]$. We measured the average Jurkat cell radius to be $5.2 \mu \mathrm{m}$. This is smaller than the radius of $7-8 \mu \mathrm{m}$ reported by other groups [20] but agrees well with the radius of $5.18 \mu \mathrm{m}$ obtained by another group [21]. Smaller cells could appear for a number of reasons, such as cell culture contamination or state in the cell cycle. Our measurement appears to be on the lower end of the reported scale. Furthermore, electropermeabilized cells can shrink to $60-70 \%$ of their initial volume (corresponding to $84-89 \%$ of their initial radius) after PEF application [22]. We observed that the radius decreased to $90 \%$ of the initial radius 1 min after the pulse and was between $93 \%$ and $94 \%$ for all times studied thereafter.

Protocol. After preparing the cell suspension, we placed a sample of control or pulsed suspension into the sample holder and measured the dielectric spectrum at 1, 5, 10, 15, and 20 min after PEF application. We periodically alternated between initially measuring the control or the pulsed sample to ensure that our observations were not artifacts due to the cells being in suspension over time. We did not observe any appreciable sedimentation over $30 \mathrm{~min}$ of measurement based on the control's cell suspension conductivity changes [17]. Details on the analysis of the real signal and subsequent correction for electrode polarization, suspension conductivity, and the two-shell model are provided elsewhere [10].

\section{Results}

Fig. 2 shows $\varepsilon_{\mathrm{m}}$ and $\sigma_{\mathrm{m}}$ versus time after PEF application. The permittivity, and thus the capacitance, of the cell membrane of pulsed Jurkat cells was consistently lower than that of the control. To determine the statistical significance of this and other differences, we conducted Student's $t$-test [23]. One minute after the pulse, the $t$-test probability $(P)$ for $\varepsilon_{\mathrm{m}}$ is 0.057 , meaning that the difference is borderline statistically significant $(P<0.05$ for statistical significance). For all other times, the difference is not statistically significant $(0.09<P<0.21)$. For $\sigma_{\mathrm{m}}$, the difference between the control and pulsed cells after $1 \mathrm{~min}$ is not statistically significant $(P=0.58)$. The difference between $\sigma_{\mathrm{m}}$ for the control and pulsed cells at all other points is statistically significant $(P<0.01)$.

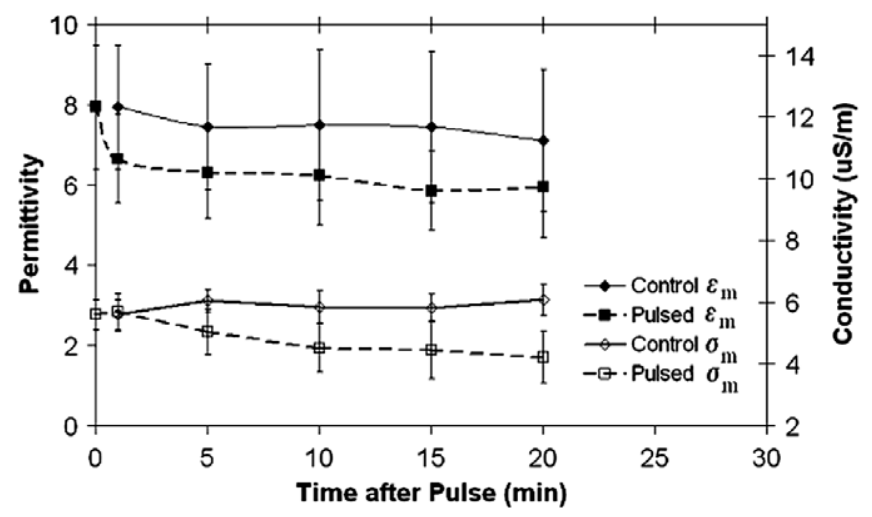

Fig. 2. Cell membrane permittivity $\left(\varepsilon_{\mathrm{m}}\right)$ and conductivity $\left(\sigma_{\mathrm{m}}\right)$ versus time after nsPEF application. The results are based on fitting experimental data from five control and nine pulsed samples to the two-shell model. Error bars are calculated using standard deviation.

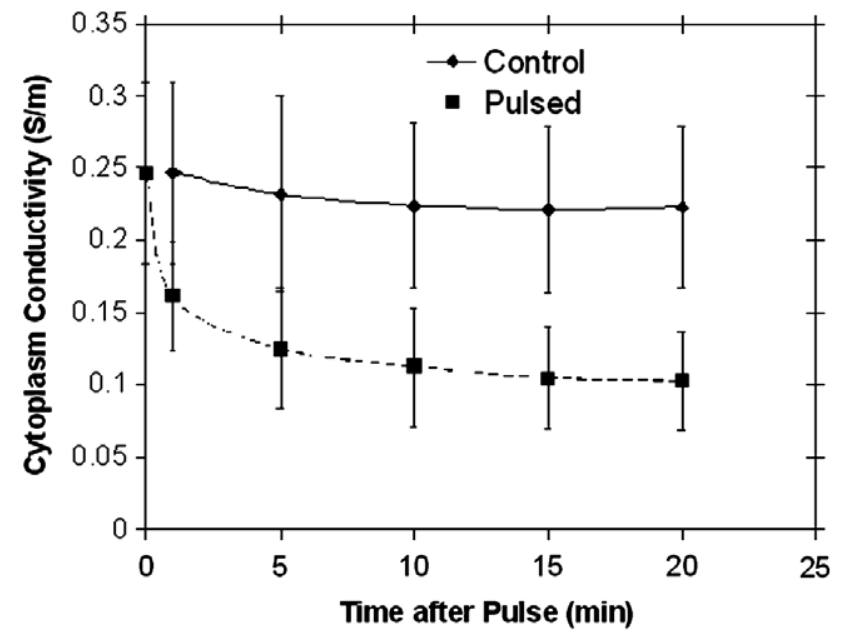

Fig. 3. Cytoplasm conductivity $\left(\sigma_{\mathrm{cp}}\right)$ versus time after nsPEF application. The electric pulses cause $\sigma_{\mathrm{cp}}$ to decrease, indicating ion transport from inside the cell to the external media. The results are based on fitting experimental data from five control and nine pulsed samples to the twoshell model. Error bars are calculated using standard deviation.

Fig. 3 shows $\sigma_{\mathrm{cp}}$ as a function of time after PEF application. The cytoplasm conductivity decreased dramatically and significantly $(P<0.01)$ within 1 min after PEF-application. From $1 \mathrm{~min}$ to $20 \mathrm{~min}$ after PEF-application, $\sigma_{\mathrm{cp}}$ of the pulsed cells decreased from $0.16 \mathrm{~S} / \mathrm{m}$ to $0.10 \mathrm{~S} / \mathrm{m}$ while the control remained essentially constant $(0.25 \mathrm{~S} / \mathrm{m}$ to $0.22 \mathrm{~S} / \mathrm{m})$. The difference between $\sigma_{\mathrm{cp}}$ of the control and pulsed cells was statistically significant $(P<0.01)$ for all times.

The results for $\varepsilon_{\mathrm{ne}}$ and $\sigma_{\mathrm{ne}}$ obtained from TDDS and fitting with the two-shell model were inconclusive concerning a PEF-induced effect are not shown.

Fig. 4 shows $\sigma_{\text {np }}$ as a function of time after PEF application. As with $\sigma_{\mathrm{cp}}, \sigma_{\mathrm{np}}$ for the pulsed cells decreased signif-

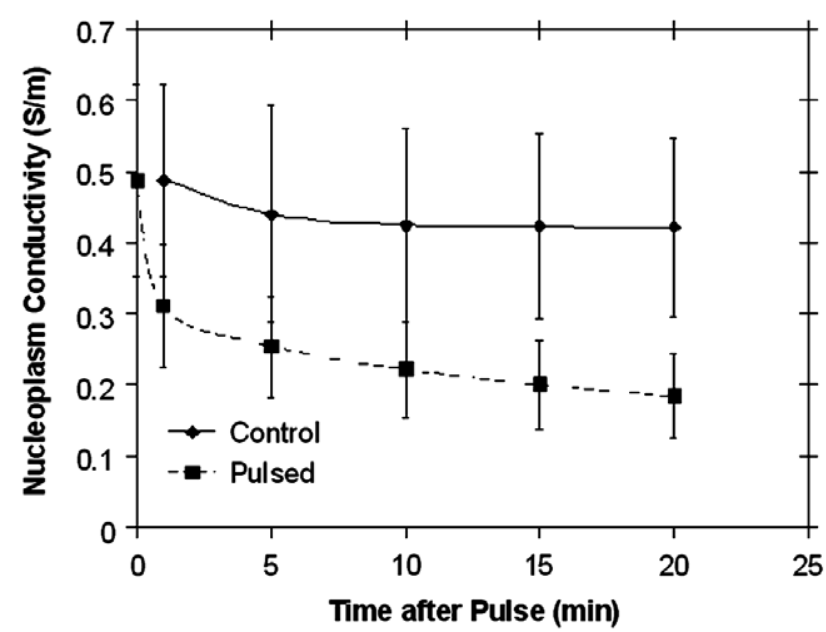

Fig. 4. Nucleoplasm conductivity $\left(\sigma_{\mathrm{np}}\right)$ versus time after nsPEF application. The electric pulses cause $\sigma_{\text {np }}$ to decrease, indicating that ions are exiting the nucleus. The results are based on fitting experimental data from five control and nine pulsed samples to the two-shell model. Error bars are calculated using standard deviation. 
icantly $(P<0.05)$ within 1 min of PEF application from $0.49 \mathrm{~S} / \mathrm{m}$ to $0.31 \mathrm{~S} / \mathrm{m}$. From $1 \mathrm{~min}$ to $20 \mathrm{~min}, \sigma_{\mathrm{np}}$ decreased from $0.31 \mathrm{~S} / \mathrm{m}$ to $0.18 \mathrm{~S} / \mathrm{m}$ for the pulsed cells and only from $0.48 \mathrm{~S} / \mathrm{m}$ to $0.42 \mathrm{~S} / \mathrm{m}$ for the control cells. The difference between the control and pulsed cells was statistically significant $(P<0.01)$ for all times beyond $5 \mathrm{~min}$.

As with previous results for lymphocytes, $\sigma_{\mathrm{np}}$ differs from $\sigma_{\mathrm{cp}}$ by a factor $1.9-2.0$ for the control $[13,14]$. The ratio between $\sigma_{\mathrm{np}}$ and $\sigma_{\mathrm{cp}}$ remained between 1.8 and 2.0 after pulsing.

\section{Discussion}

The overall results summarized in Fig. 2 through Fig. 4 are consistent with the range of experimental values for eukaryotic cells obtained from the two-shell model [24]. The values we obtained for $\sigma_{\mathrm{m}}, \sigma_{\mathrm{cp}}, \sigma_{\mathrm{np}}, \varepsilon_{\mathrm{ne}}$, and $\sigma_{\text {ne }}$ (data not shown for $\varepsilon_{\text {ne }}$ and $\sigma_{\text {ne }}$ ) are lower than those obtained for other malignant $\mathrm{T}$-cell lines while $\varepsilon_{\mathrm{m}}$ is in line with these results $[10,11]$. These differences could be attributed to the cell lines we are considering here, different cell preparation techniques, or the number of cell passages; however, our results were consistent with each other and the standard deviations are similar to those obtained in Ref. [10].

When electroporation occurs, the cell membrane becomes more permeable to molecules and ions that are sufficiently small to traverse the pores. The prevalent theory is that the cell membrane must be charged to a voltage in excess of $1 \mathrm{~V}$ for a sufficient period of time to generate a pore [25]. Furthermore, the cell can recover (i.e. the pores reseal) as long as the pore radius remains below a certain critical radius, $r_{\text {crit }}[25,26]$. While $10 \mathrm{~ns}$ pulses are generally too short to induce electroporation [4], applying multiple pulses charges the cell membrane more, increasing the likelihood of exceeding $r_{\text {crit }}$ [26]. Using a lower conductivity buffer also reduces the electroporation threshold for nsPEFs [27]. Applying a single $11 \mathrm{~ns}, 160 \mathrm{kV} / \mathrm{cm}$ pulse to a cell suspension with conductivity of approximately $0.1 \mathrm{~S} / \mathrm{m}$ resulted in slightly less than $10 \%$ of the cells taking in propidium iodine [27]. Here, we applied five pulses with similar parameters, likely increasing the fraction of cells electropermeabilized.

Figs. 3 and 4 show the behavior of cytoplasm and nucleoplasm conductivity as a function of time. The control value of $\sigma_{\mathrm{cp}}$ falls within the range of values $\left(0.033<\sigma_{\mathrm{cp}}<1.1 \mathrm{~S} / \mathrm{m}\right)$ stated by Ermolina et al. [24]. However, it is lower than those stated elsewhere in the literature [28]. One must consider that these analyses used a singleshell model, so they considered the nucleus and cytoplasm together, which would make $\sigma_{\mathrm{cp}}$ larger than in the two-shell model [10].

Our results suggest that this pulse combination with the low conductivity buffer induces noticeable ion transport, as illustrated by a $14 \%$ rise in cell suspension conductivity, which is comparable to the conductivity rise observed for a single $50 \mu$ s pulse of the same energy [17]. Figs. 3 and 4 show that $\sigma_{\mathrm{cp}}$ and $\sigma_{\mathrm{np}}$ both decreased dramatically and sig- nificantly following nsPEF application, indicating pulseinduced ion transport. This is consistent with preliminary fluorescent experiments where applying five $10 \mathrm{~ns}$, $150 \mathrm{kV} / \mathrm{cm}$ pulses or a single $10 \mathrm{~ns}, 300 \mathrm{kV} / \mathrm{cm}$ pulse to Jurkat cells causes a large drop in $\mathrm{Na}+$ and $\mathrm{K}+$ fluorescence [17]. Both $\sigma_{\mathrm{cp}}$ and $\sigma_{\mathrm{np}}$ continued to decrease for up to $10 \mathrm{~min}$ after the pulse, indicating ongoing ion transport and that full membrane recovery may take on the order of $10 \mathrm{~min}$ to occur. The maintenance of the factor of two ratio between $\sigma_{\mathrm{cp}}$ and $\sigma_{\mathrm{np}}$ suggests that the nuclear envelope remained a diffusion barrier and that its ion channels could be maintaining this equilibrium [29].

While cell suspension conductivity, $\sigma_{\mathrm{cp}}$, and $\sigma_{\mathrm{np}}$ clearly show that ions are leaving the nucleoplasm and cytoplasm $\left(\sigma_{\mathrm{cp}} \sim 0.23 \mathrm{~S} / \mathrm{m}\right)$ for the surrounding buffer $(\sigma$ $\sim 0.10 \mathrm{~S} / \mathrm{m}$ ), the resulting changes in $\sigma_{\mathrm{m}}$ (Fig 4) are less apparent. Because electroporation raises the permeability of the cell membrane to all molecules and ions, one would expect $\sigma_{\mathrm{m}}$ to rise after the pulse, as observed in electroporation experiments [30] and in experiments by our group for Jurkat cells exposed to a $6 \mathrm{kV} / \mathrm{cm}$, $300 \mathrm{~ns}$ pulse (K.H. Schoenbach, personal communication). However, Fig 2 shows that the conductivity actually does not change significantly $1 \mathrm{~min}$ after the pulse and actually decreases after that.

Since the largest portion of the change in $\sigma_{\mathrm{cp}}$ and $\sigma_{\mathrm{np}}$ has already occurred within 5 min after pulse application, the majority of ion transport has occurred during this time. The subsequent leveling of these conductivities suggests membrane recovery. The preliminary increase in $\sigma_{\mathrm{m}}$ may have occurred and already recovered prior to the first TDDS measurement with the membrane mostly recovered in this timeframe, as suggested by modeling [26]. However, one would anticipate that $\sigma_{\mathrm{m}}$ would remain at the control value following recovery.

The long-term decrease in $\sigma_{\mathrm{m}}$ could arise due to a change in ion channel structure and/or function. PEFs could damage ion channels. While not yet investigated for multiple nsPEFs, ionizing radiation dramatically and rapidly (within $\ll 1 \mathrm{~s}$ ) lowers $\sigma_{\mathrm{m}}$ by inactivating gramicidin channels and reducing the formation rate of open gramicidin channels [31]. Another possibility could be use dependence, a "blockade" of effected ion channels that occurs due to repeated cycles in membrane potential [32].

Fig. 3 shows $\varepsilon_{\mathrm{m}}$ as a function of time for both control and pulsed samples. For the control, $\varepsilon_{\mathrm{m}}$ varied between 6 and 8 , corresponding to a membrane capacitance, $C_{\mathrm{m}}$, between $7.6 \mathrm{mF} / \mathrm{m}^{2}$ and $10 \mathrm{mF} / \mathrm{m}^{2}\left(C_{\mathrm{m}}=\varepsilon_{\mathrm{m}} \varepsilon_{0} / d\right.$, where $d$ is the membrane thickness). The typical value for the isotonic $C_{\mathrm{m}}$ of Jurkat cells is approximately $14 \mathrm{mF} / \mathrm{m}^{2}$ [20]. However, our measurements are within the typical range for $\mathrm{T}$ cells, which Pethig et al. lists as $6-15 \mathrm{mF} / \mathrm{m}^{2}$ [21].

Multiple nsPEFs caused $\varepsilon_{\mathrm{m}}$ to decrease, but only the decrease $1 \mathrm{~min}$ after the pulse was borderline statistically significant. This could mean that an nsPEF-induced effect on $\varepsilon_{\mathrm{m}}$ occurred on a timescale shorter than measurable by TDDS. Subsequent time points had little to no 
statistical significance, indicating negligible long-term nsPEF-induced effect.

Because nsPEFs interact with intracellular organelles [4], one would anticipate strong effects on $\varepsilon_{\mathrm{ne}}$ and $\sigma_{\mathrm{ne}}$; however, the impact of nsPEFs upon both $\varepsilon_{\text {ne }}$ and $\sigma_{\text {ne }}$ on the timescales of interest was inconclusive. This could be due to measurement system sensitivity, the timescale on which nuclear effects occur (modeling suggests that nuclear envelope voltage decays to 0 within $300 \mathrm{~ns}$ [26]), or the large number of pores already present in the nuclear envelope masking the permeabilization.

These results indicate that multiple nsPEFs alter the structure and function of cell structures. Adapting the TDDS technique to allow real-time measurement (on the order of $\mu \mathrm{s}$ ) of the dielectric spectra would permit a more direct study of pulse-induced effects. Improving measurement sensitivity may permit determination of the importance and significance of slight changes of the nuclear envelope.

\section{Acknowledgments}

This work was supported in part by an Air Force Office of Scientific Research (AFOSR) DOD MURI grant on Subcellular Response to Narrow Band and Wide Band Radio Frequency Radiation, administered by Old Dominion University, and in part by an AFOSR grant on bioinspired concepts. One author (ALG) was supported by a National Defense Science and Engineering Graduate Fellowship sponsored by the Department of Defense. G.C. is grateful to the University of Southampton for granting him study leave. The authors appreciate the assistance provided by Wentia Ford, Rachael Shevin, Elizabeth Coon, and Kris Ball. The authors also thank David Jordan, Michael Mayer, Uwe Pliquett, Michael Uhler, Ron Gilgenbach, and Y.Y. Lau for many stimulating discussions. We are also grateful for insight into data analysis procedures and software provided by Yoshihito Hayashi, Irina Ermolina, and Yuri Feldman.

\section{References}

[1] J. Teissie, N. Eynard, B. Gabriel, M.P. Rols, Electropermeabilization of cell membranes, Adv. Drug Del. Rev. 35 (1999) 3-19.

[2] K.H. Schoenbach, R.P. Joshi, R.H. Stark, F.C. Dobbs, S.J. Beebe, Bacterial decontamination of liquids with pulsed electric fields, IEEE Trans. Diel. Elec. Insul. 7 (2000) 637-645.

[3] D. Rabussay, N.B. Dev, J. Fewell, L.C. Smith, G. Widera, L. Zhang, Enhancement of therapeutic drug and DNA delivery into cells by electroporation, J. Phys. D: Appl. Phys. 36 (2003) 348-363.

[4] K.H. Schoenbach, R.P. Joshi, J.F. Kolb, N. Chen, M. Stacey, P.F. Blackmore, E.S. Buescher, S.J. Beebe, Ultrashort electrical pulses open a new gateway into biological cells, Proc. IEEE 92 (2004) 1122 1137

[5] S.J. Beebe, J. White, P.F. Blackmore, Y. Deng, K. Somers, K.H. Schoenbach, Diverse effects of nanosecond pulsed electric fields on cells and tissues, DNA Cell Biol. 22 (2003) 785-796.

[6] E.S. Buescher, R.R. Smith, K.H. Schoenbach, Submicrosecond intense pulsed electric field effects on intracellular free calcium: mechanisms and effects, IEEE Trans. Plasma Sci. 32 (2004) 15631572.

[7] S.J. Beebe, P.M. Fox, L.J. Rec, K. Somers, R.H. Stark, K.H. Schoenbach, Nanosecond pulsed electric fields (nsPEF) effects on cells and tissues: Apoptosis induction and tumor growth inhibition, IEEE Trans. Plasma Sci. 30 (2002) 286-292.

[8] S.J. Beebe, P.M. Fox, L.J. Rec, E.L. Willis, K.H. Schoenbach, Nanosecond, high-intensity pulsed electric fields induce apoptosis in human cells, Fed. Am. Soc. Exp. Biol. J. 17 (2003) 1493-1495.

[9] R.P. Joshi, Q. Hu, K.H. Schoenbach, Modeling studies of cell response to high-intensity electric fields-implications for intracellular manipulation, IEEE Trans. Plasma Sci. 32 (2004) 1677-1686.

[10] Y. Polevaya, I. Ermolina, M. Schlesinger, B.-Z. Ginzburg, Y. Feldman, Time domain dielectric spectroscopy study of human cells II. Normal and malignant white blood cells, Biochim. Biophys. Acta 1419 (1999) 257-271.

[11] Y. Feldman, I. Ermolina, Y. Hayashi, Time domain dielectric spectroscopy study of biological systems, IEEE Trans. Diel. Elec. Insul. 10 (2003) 728-753.

[12] A. Irimajiri, Y. Doida, T. Hanai, A. Inouye, Passive electrical properties of cultured murine lymphoblast (L5178Y) with reference to its cytoplasmic membrane, nuclear envelope, and intracellular phases, J. Membr. Biol. 38 (1978) 209-232.

[13] J. Gisma, P. Marszalek, U. Loewe, T.Y. Tsong, Dielectrophoresis and electrorotation of neurospora slime and murine myeloma cells, Biophys. J. 60 (1991) 749-760.

[14] Y. Feldman, A. Andrianov, E. Polygalov, I. Ermolina, G. Romanychev, Y. Zuev, B. Milgotin, Time domain dielectric spectroscopy: An advanced measuring system, Rev. Sci. Instrum. 67 (1996) 3208-3216.

[15] F. Bordi, C. Cametti, J.S. Tan, D.C. Boris, W.E. Krause, N. Pluckateesak, R.H. Colby, Determination of polyelectrolyte charge and interaction with water using dielectric spectroscopy, Macromolecules 35 (2002) 7031-7038.

[16] A.L. Garner, N. Chen, J. Yang, J. Kolb, R.J. Swanson, K.C. Loftin, S.J. Beebe, R.P. Joshi, K.H. Schoenbach, Time domain dielectric spectroscopy measurements of HL-60 cell suspensions after microsecond and nanosecond electrical pulses, IEEE Trans. Plasma Sci. 32 (2004) 2073-2084.

[17] G. Chen, N. Chen, A.L. Garner, J. Kolb, R.J. Swanson, S. Beebe, R.P. Joshi, K.H. Schoenbach, Conductivity in Jurkat cell suspension after ultrashort electric pulsing, in: Proceedings of the 3rd International Workshop on Biological Effect of EMFs, Kos, Greece, vol. 1, 2004 , pp. 56-65.

[18] J.F. Kolb, S. Kono, K.H. Schoenbach, Nanosecond pulsed electric field generators for the study of subcellular effects, Bioelectromagnetics 27 (2006) 172-187.

[19] Y. Feldman, E. Polygalov, I. Ermolina, Yu. Polevaya, B. Tsentsiper, Electrode polarization correction in time domain dielectric spectroscopy, Meas. Sci. Technol. 12 (2001) 1355-1364.

[20] M. Kiesel, R. Reuss, J. Endter, D. Zimmermann, H. Zimmermann, R. Shirakashi, E. Bamberg, U. Zimmermann, V.L. Sukhorukov, Swelling-activated pathways in human T-lymphocytes studied by cell volumetry and electrorotation, Biophys. J. 90 (2006) 4720-4729.

[21] R. Pethig, V. Bressler, C. Carswell-Crumpton, Y. Chen, L. FosterHaje, M.E. Garcia-Ojeda, R.S. Lee, G.M. Lock, M.S. Talary, K.M. Tate, Dielectrophoretic studies of the activation of human $\mathrm{T}$ lymphocytes using a newly developed cell profiling system, Electrophoresis 23 (2002) 2057-2063.

[22] R. Shirakashi, V.L. Sukhorukov, I. Tanasawa, U. Zimmermann, Measurement of the permeability and resealing time constant of the electroporated mammalian cell membranes, Int. J. Heat Mass Transfer 47 (2004) 4517-4524.

[23] S.A. Glantz, Primer of Biostatistics, 4th Ed., McGraw-Hill, New York, 1997, 65-105.

[24] I. Ermolina, Yu. Polevaya, Yu. Feldman, Analysis of dielectric spectra of eukaryotic cells by computer modeling, Eur. Biophys. J. 29 (2000) 141-145. 
[25] J.C. Weaver, Yu.A. Chizmadzhev, Theory of electroporation: a review, Bioelectrochem. Bioenergy 41 (1996) 135-160.

[26] R.P. Joshi, K.H. Schoenbach, Mechanism for membrane electroporation irreversibility under high-intensity, ultrashort electrical pulse conditions, Phys. Rev. E 66 (2002) 052901.

[27] K.J. Müller, V.L. Sukhorukov, U. Zimmermann, Reversible electropermeabilization of mammalian cells by high-intensity, ultra-short pulses of submicrosecond duration, J. Membrane Biol. 184 (2001) $161-170$.

[28] Y. Huang, X.-B. Wang, P.R.C. Gascoyne, F.F. Becker, Membrane dielectric responses of human T-lymphocytes following mitogenic stimulation, Biochim. Biophys. Acta 1417 (1999) 51-62.
[29] M. Mazzanti, Ion permeability of the nuclear envelope, News Physiol. Sci. 13 (1998) 44-50.

[30] M. Schmeer, T. Seipp, U. Pliquett, S. Kakorin, E. Neumann, Mechanism for the conductivity changes caused by membrane electroporation of $\mathrm{CHO}$ cell-pellets, Phys. Chem. Chem. Phys. 6 (2004) 5564-5574.

[31] G. Stark, The effect of ionizing radiation on lipid membranes, Biochim. Biophys. Acta 1071 (1991) 103-122.

[32] J.A.H. Verheugen, H.P.M. Vijverberg, M. Oortgiesen, M.D. Cahalan, Voltage-gated and $\mathrm{Ca}^{2+}$-activated $\mathrm{K}^{+}$channels in intact human T lymphocytes, J. Gen. Physiol. 105 (1995) 765-794. 